\title{
Ultrasound guided erector spinae plane block in thoracic trauma
}

\author{
Achenbach, J., Schott.M.
}

\section{INTRODUCTION}

Acute pain is a common occurrence on any intensive care unit as a result of trauma and major emergency surgery. In elective cases continuous neuraxial blocks are a cornerstone of multimodal analgesia. In an emergency setting, the issues of consent, coagulopathies and sepsis often preclude the use of such procedures. Additionally, performing neuraxials blocks in patients that are intubated and sedated is challenging. Neurological complications are also difficult to assess. Uncontrolled pain will increase sedation requirements, the likelyhood of respiratory complications and long term morbidity as well as chronic pain. These issues become especially apparent in thoracic trauma and emergency thoracotomy which carry an increased of respiratory failure and persistent pain. We observe promising results with the erector spinae plane block (ESP) which is easy to perform with a steep learning curve.

We present a case of thoracic trauma, in which the ESP has been successfully employed to supplement general anaesthesia and alleviate postoperative pain which resulted in improved respiratory function and mobilisation.

\section{Case: Serial Rib Fracture And PNEUMOTHORAX}

We report the case of a 40 -year-old male who fell $16 \mathrm{ft}$ from a ladder doing yardwork. He presented with a haematopneumothorax and serial dislocated rib fracture (5-9) on the left side. With concurrend atelectasis an emergent VATS was scheduled. His past medical history consists of asthma and heavy smoking. Due to the acute setting of the situation, informed consent for a central neuraxial block was considered insufficient. Out of concern for postoperative respiratory compromise however, the patient agreed to the implementation of an ESP block with placement of a catheter for postoperative analgesia using an 18G Tuohy needle. Visualization was achieved using a Philips CX50 ultrasound system and linear probe. After routine induction of anaesthesia and maintenance with propofol and remifentanil, the patient undervent uncomplicated surgery. Postoperatively, respiratory mechanics and pain scores allowed further treatment on the normal ward. Mobilisation could be achieved early and only non-opiod analgesics were needed in addition to intermittent $15 \mathrm{ml}$ boluses of ropivacain $0,2 \%$ via the ESP catheter. The catheter was removed on postoperative day 3 . No adverse side effects were noted.

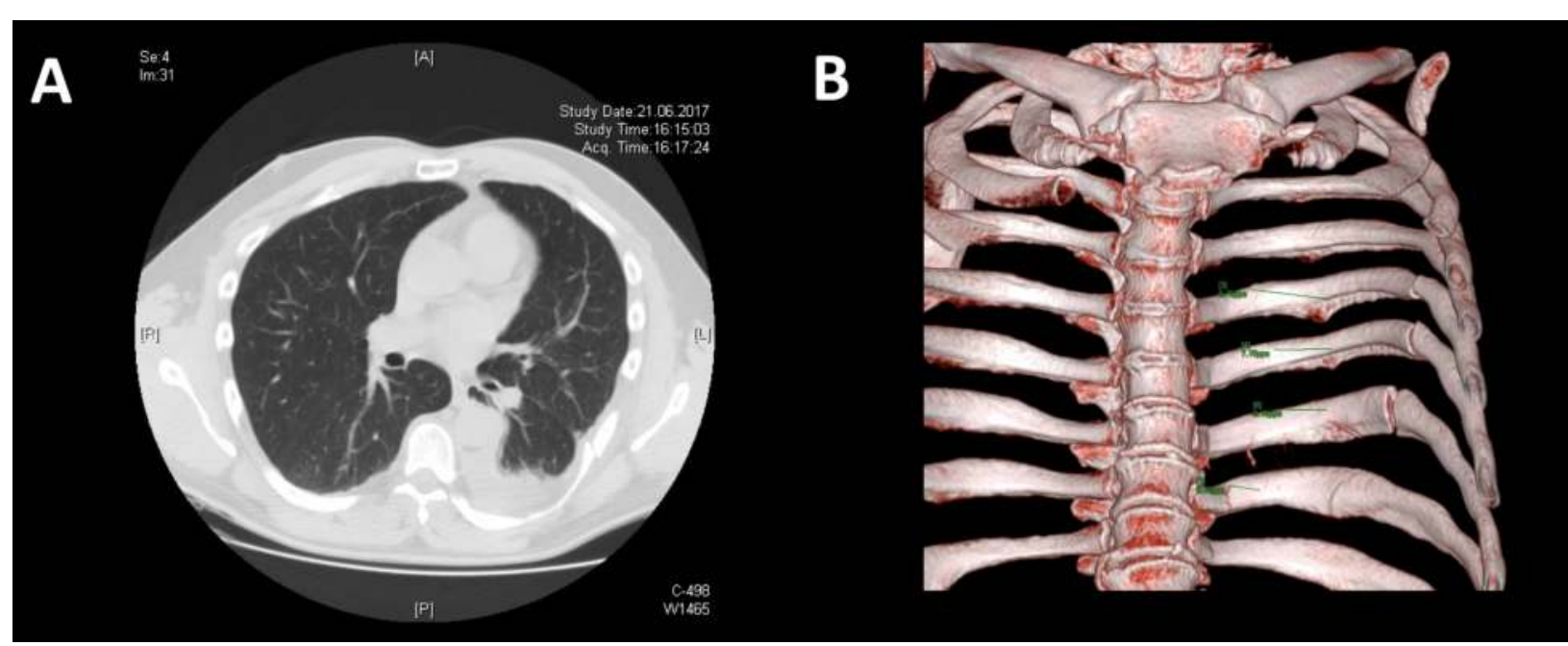

Fig. 1. Native CT (A) and 3D-reconstruction (B)

\section{TECHNIQUE}

The erector spinae block was first described by Forero et al in 2016 (1) and further tested in ventral hernia repair by Chin and colleagues. A modification of the technique is described as the mid-point transverse process to pleura (MTP) block by Costache et al(2). Both techniques aim to access the paravertebral space through a more superficial injection site compared with traditional paravertebral appraoches. The goal is to minimize complications like vascular puncture and perforation of the pleura. Chin an colleagues target the lateral tip of the transverse process (TP) while advancing the needle in plane in a cephalad-to-caudad direction at the level of T5. A successful application is signified by lifting of the erector spinae muscle off the transverse processes under ultrasound guidance. Costache describes the injection site as the midpoint between the TP and pleura where he observes pleural displacement similar to a traditional paravertebral block as well as lifting of the erector spinae muscle.

Due to the continuous nature and fascial surrounding of the erector spinae muscle it is most likely possible to use this technique on different levels for different indications as suggested by an small number of case reports (i.e. analgesia for hip arthroplasty after block performance at the level of L4(3). This is most likely made possible by the paravertebral space not being as distinct an entity as it was once believed.

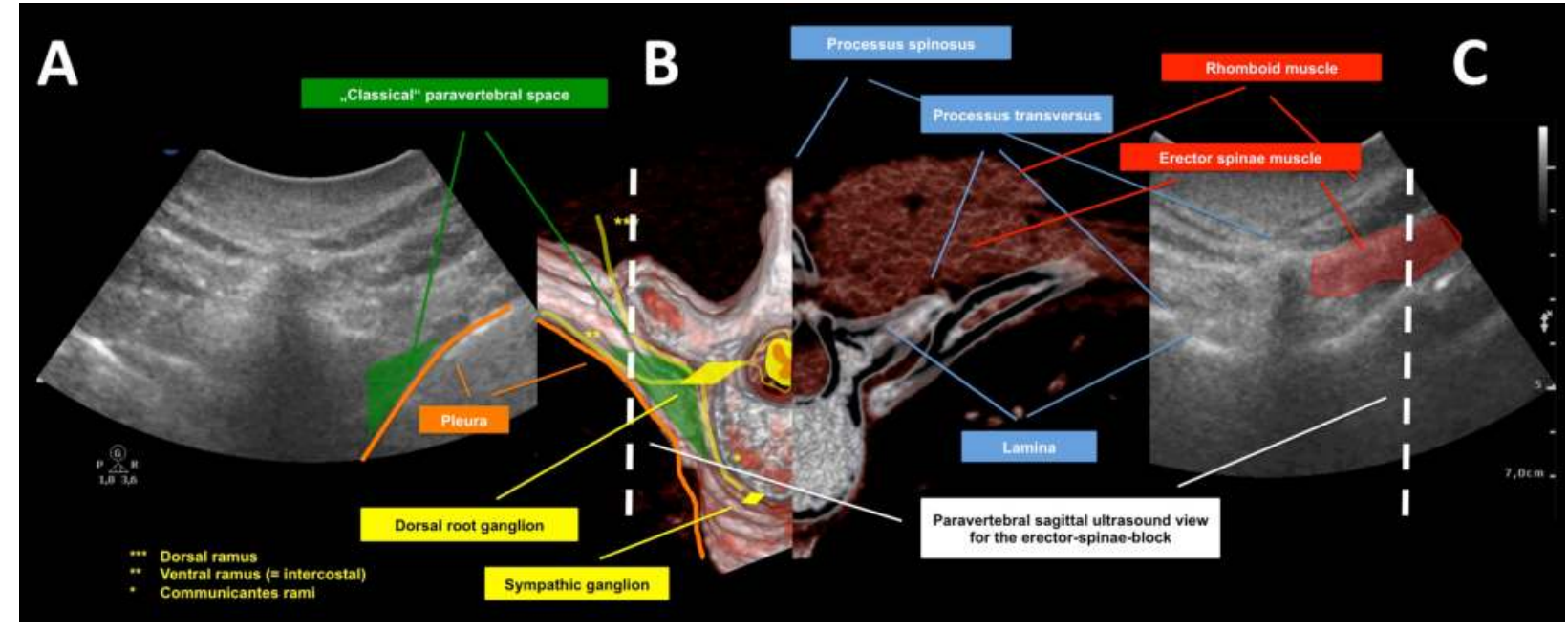

Fig. 2. $A, C$ : transverse sections with $C$ at the level of and $A$ just below a transverse process; $\mathrm{B}$ : anatomical schematics within a 3D-reconstruction

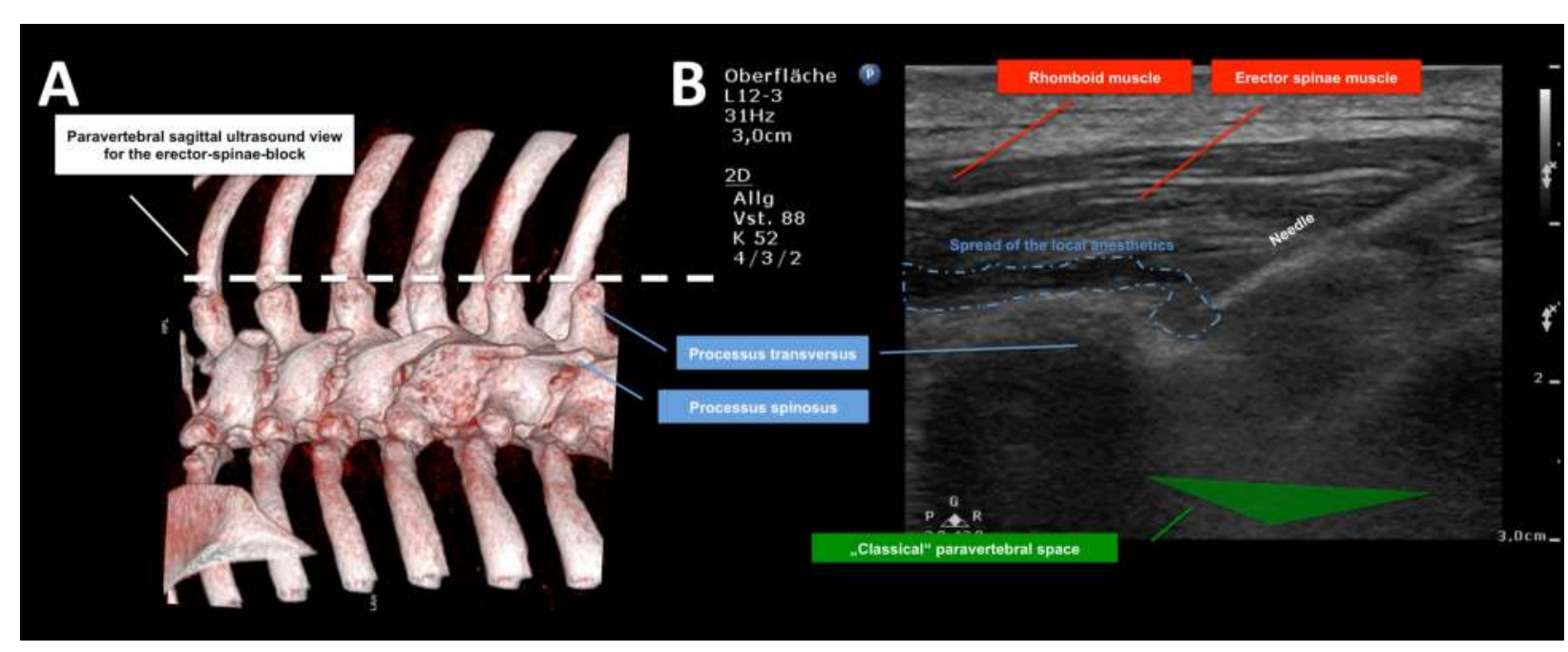

Fig. 3. A: 3D-reconstruction, probe placement for acquisition of image B; $B$ : local anaesthetic deposit between erector spinae muscle and transverse process

\section{Discussion}

Over the last few years the serratus anterior plane block among others have been utilized for pain relieve in rib fractures, placing chest drains and surgery of the lateral and anterior chest wall. However, in case of posterior rib fractures block failures have been described. The anatomical basis of the ESP block's efficacy in this setting is the closeness to the dorsal rami of the spinal nerves which innervate the posterior part of the chest wall(4). The dorsal rami transverse the erector spinae plane and are effectively covered by local anaesthetic. Additionally, the LA spreads anteriorly and cephalad to caudad (supposedly through gaps in the costoransverse ligaments) into the paravertebral space(1). This could explain the observed anaesthesia of the lateral and anterior chest wall.

The ESP block is a simple, easy and safe to perform technique with numerous potential applications in emergency and elective settings(1-5). It helps to avoid many of the risks of central neuraxial techniques. Systematic studies are needed to reliably assess block spread, efficacy and safety of catheter placement and optimal dosages of local anaesthetics. A direct comparison with established techniques is also warranted.

Dr. Johannes Achenbach, DESA Department of Anaesthesiology and Intensive Care Medicine, Medizinische Hochschule Hannover, Hannover, Germany

Dr. Martin Schott, DESA Department of Anaesthesiology, Intensive Care Medicine, Pain Therapy and Emergency Rescue Medicine, Klinikum Nordstadt, Hannover, Germany

\section{REFERENCES}

1. Forero M, Adhikary SD, Lopez H, Tsui C, Chin KJ. The Erector Spinae Plane Block: A Novel Analgesic Technique in Thoracic Neuropathic Pain. Reg Anesth Pain Med. 2016;41:621-627

2. Costache I, de Neumann L, Ramnanan CJ et al. The mid-point transverse process to pleura (MTP) block: a new end-point for thoracic paravertebral block. Anaesthesia. 2017;72:1230-1236.

3. Tulgar S, Senturk O. Ultrasound guided Erector Spinae Plane block at L-4 transverse process level provides effective postoperative analgesia for total hip arthroplasty. Journal of Clinical Anesthesia. 2018;44:68.

4. Luftig J, Mantuani D, Herring AA, Dixon B, Clattenburg E, Nagdev A. Successful emergency pain control for posterior rib fractures with ultrasound-guided erector spinae plane block. Am J Emerg Med. 2017

5. Hernandez MA, Palazzi L, Lapalma J, Forero M, Chin KJ. Erector Spinae Plane Block for Surgery of the Posterior Thoracic Wall in a Pediatric Patient. Reg Anesth Pain Med. 2018;43:217-219. 